Annals of Pure and Applied Mathematics

Vol. 18, No. 1, 2018, 99-112

ISSN: 2279-087X (P), 2279-0888(online)

Published on 19 September 2018

www.researchmathsci.org

DOI: http://dx.doi.org/10.22457/apam.v18n1a15

Annals of

Pure and Applied

Mathematics

\title{
Asymptotic Solutions of Coupled Spring Systems with Cubic Nonlinearity using Homotopy Perturbation Method
}

\author{
Md. Abdul Alim ${ }^{1}$, M. Abul Kawser ${ }^{2}$ and Md. Mizanur Rahman ${ }^{3}$ \\ ${ }^{1}$ Department of Electrical and Electronic Engineering \\ Pundra University of Science and Technology, Bogura-5800, Bangladesh. \\ ${ }^{1}$ E-mail: alim.math.iu@gmail.com \\ ${ }^{2,3}$ Department of Mathematics, Faculty of Applied Science and Technology \\ Islamic University, Kushtia-7003, Bangladesh. \\ 22E-mail: kawserma@yahoo.com; ${ }^{3}$ E-mail: mmrmathsiubd@gmail.com \\ ${ }^{3}$ Corresponding author
}

Received 9 August 2018; accepted 15 September 2018

Abstract. In recent years, many analytical and numerical methods have emerged which are being used to obtain approximate solutions of a wide range of problems arising in mathematical modeling of linear and nonlinear physical and engineering problems. In this paper we study the application of the homotopy perturbation method (HPM) to obtain analytical approximate solutions of the nonlinear differential equations which model a coupled spring system with and without damping and external driving force. The application of the method is found to be justified by a good agreement between the results of HPM and the corresponding numerical ones obtained by using Mathematica 9 .

Keywords: Homotopy perturbation method, Coupled spring equations, Damping, External force

AMS Mathematics Subject Classification (2010): 34E10, 37M99

\section{Introduction}

From the mathematical point of view, most of the real world physical and engineering problems are modeled as differential equations. While standard solution procedures exist for linear differential equations, nonlinear equations are rather difficult to solve, and in some cases, it is virtually impossible to find exact solutions. Mathematicians are in a constant search of new techniques to find analytically exact or approximate solutions for nonlinear ordinary and partial differential equations which model diverse fields of science and engineering. Some of the recently developed and popular methods used to find approximate solutions to nonlinear problems are the homotopy perturbation method (HPM) [1-7], the variational iteration method (VIM) [8-10], and the Adomian decomposition method $[11,12]$. The homotopy perturbation method, introduced by the Chinese mathematician Dr. Ji Huan He in 1998, has come to be accepted as an elegant tool in the hands of researchers looking for simple yet highly effective solutions to 
Md. Abdul Alim, M. Abul Kawser and Md. Mizanur Rahman

complicated problems in many diverse areas of science and technology. It has been employed to solve a large variety of linear and nonlinear problems and found to provide highly accurate solutions in comparison with numerical techniques. In [13] and [14] He applied the HPM for solving nonlinear boundary value problem and Blasius differential equation, respectively. Ganji and Rafei used the HPM in [15] to obtain solitary wave solutions for a generalized nonlinear Hirota-Satsuma coupled KdV partial differential equations. This HPM has also been successfully applied to problems relating to the Laplace equation [16], heat radiation equations [17], nonlinear dispersive $K(\mathrm{mp})$ equations [18], nonlinear integral equations [19], nonlinear heat conduction and convection equations [20], nonlinear Schrödinger equations [21], nonlinear oscillators [22], nonlinear wave equations [23], nonlinear chemistry problems [24], and to other fields [25-32]. The HPM yields a very rapid convergence of the solution series in most cases, usually only a few iterations leading to very accurate solutions.

The aim of this article is to extend the application of the He's HPM to solve a system of nonlinear ordinary differential equations which give a mathematical model of coupled spring systems [33]. Mathematica 9.0 software has been used for computing and testing the accuracy of the analytical approximate HPM solutions compared with the numerical solutions.

\section{Formulation of the Problem}

The coupled spring system we study consists of two springs and two weights. One spring, having spring constant $k_{1}$ is attached to the ceiling and a weight of mass $m_{1}$ is attached to the lower end of this spring. To this weight, a second spring is attached having spring constant $k_{2}$. To the bottom of this second spring, a weight of mass $m_{2}$ is attached and the entire system appears as illustrated in Figure 1. Allowing the system to come to rest in equilibrium, we measure the displacement of the centre of mass of each weight from equilibrium as a function of time, and denote these measurements by $x_{1}(t)$ and $x_{2}(t)$ respectively.

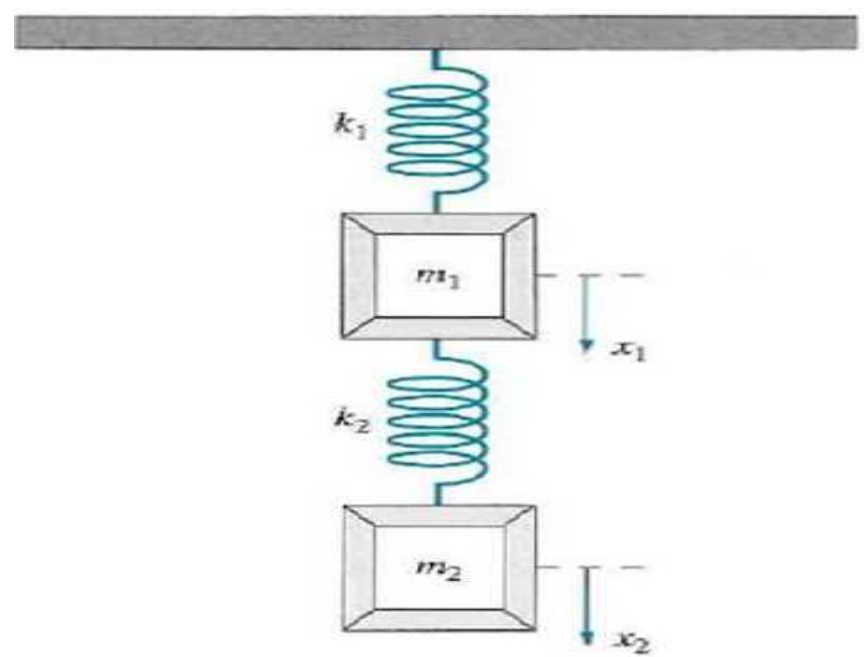

Figure 1: The coupled spring system. 


\section{Asymptotic Solutions of Coupled Spring Systems with Cubic Nonlinearity using Homotopy Perturbation Method}

Assuming Hooke's Law, under the assumption of small oscillations, the restoring forces are of the form $-k_{1} l_{1}$ and $-k_{2} l_{2}$ where $l_{1}$ and $l_{2}$ are the elongations (or compressions) of the two springs. Since the upper mass is attached to both springs, there are two restoring forces acting upon it: an upward restoring force $-k_{1} x_{1}$ exerted by the elongation (or compression) $x_{1}$ of the first spring; an upward force $-k_{2}\left(x_{2}-x_{1}\right)$ from the second spring's resistance to being elongated (or compressed) by the amount of $x_{2}-x_{1}$. The second mass only 'feels' the restoring force from the elongation (or compression) of the second spring. If we assume that there are no damping forces present, then Newton's Law implies that the two equations representing the motions of the two weights are

$$
\begin{aligned}
& m_{1} x_{1}^{\prime \prime}=-k_{1} x_{1}-k_{2}\left(x_{1}-x_{2}\right) \\
& m_{2} x_{2}^{\prime \prime}=-k_{2}\left(x_{2}-x_{1}\right)
\end{aligned}
$$

where primes denote derivation with respect to time. Thus we have a pair of coupled second-order linear differential equations.

The most common type of damping encountered in beginning courses is that of viscous damping; the damping force is proportional to the velocity. The damping of the first weight depends solely on its velocity and not the velocity of the second weight, and vice versa. We assume that the damping coefficients $\delta_{1}$ and $\delta_{2}$ are small. We add viscous damping to the model by adding the term $-\delta_{1} x_{1}^{\prime}$ to the equation (1) and $-\delta_{2} x_{2}^{\prime}$ to the equation (2).

If we assume that the restoring forces are nonlinear, which are most certainly the cases of large vibrations, we can modify the model accordingly. Rather than assuming that the restoring force is of the form $-k x$ (Hooke's law), we assume the restoring force has the form $-k x+\mu x^{3}$. We add nonlinearity to the model by adding the terms $\mu_{1} x_{1}^{3}$ and $\mu_{2}\left(x_{1}-x_{2}\right)^{3}$ to the equation (1) and $\mu_{2}\left(x_{2}-x_{1}\right)^{3}$ to the equation (2). The range of motions for such nonlinear model is much more complicated than that for the corresponding linear model. An idea of this range of motions for a single spring model is given in [34]. Moreover, accuracy questions arise when solving these equations. No numerical solution can be expected to remain accurate over long time intervals. The accumulated local truncation error, algorithm error, round off error, propagation error, etc., eventually force the numerical solution to be inaccurate. This is discussed in some detail in the interesting papers by Knapp and Wagon [35], and by Fay and Joubert [36, 37].

It is a simple matter to add external forcing to the model. Indeed, we can drive each weight differently. Suppose we assume simple sinusoidal forcing of the form $F \cos \omega t$. Then the model becomes

$$
\begin{aligned}
& m_{1} x_{1}^{\prime \prime}=-\delta_{1} x_{1}^{\prime}-k_{1} x_{1}+\mu_{1} x_{1}^{3}-k_{2}\left(x_{1}-x_{2}\right)+\mu_{2}\left(x_{1}-x_{2}\right)^{3}+F_{1} \cos \omega_{1} t \\
& m_{2} x_{2}^{\prime \prime}=-\delta_{2} x_{2}^{\prime}-k_{2}\left(x_{2}-x_{1}\right)+\mu_{2}\left(x_{2}-x_{1}\right)^{3}+F_{2} \cos \omega_{2} t
\end{aligned}
$$


Md. Abdul Alim, M. Abul Kawser and Md. Mizanur Rahman

The range of motions for nonlinear forced models is quite vast. We can expect to find bounded and unbounded solutions (nonlinear resonance), periodic solutions that share the period with the forcing (called harmonic solutions) and solutions that are periodic with a period of a multiple of the driving period (called sub harmonic solutions), and steady state periodic solutions (limit cycles in the phase plane). The conditions under which these motions occur are by no means easy to state.

\section{Homotopy Perturbation Method}

To illustrate the homotopy perturbation method, we consider a general equation of the type,

$$
A(u(x))-f(r)=0, r \in \Omega
$$

With the boundary conditions $B\left(u, \frac{d u}{d x}\right)=0, r \in \Gamma$

where $A$ is the general differential operator, $B$ is the boundary operator, $\Gamma$ is the boundary of the domain $\Omega$ and $f(r)$ is a known analytical function. Generally speaking, the operator $A$ can be divided into a linear part $L$ and a nonlinear part $N$. Now equation (5) can be written as:

$$
L(u(x))+N(u(x))-f(r)=0
$$

By the homotopy perturbation method, we construct a homotopy as $v(r, p): \Omega \times[0,1] \rightarrow R$ which satisfies the following equation:

$$
H(v, p)=(1-p)\left[L(v)-L\left(u_{0}\right)\right]+p[A(v)-f(r)]=0
$$

where $p \in[0,1]$ is an embedding parameter and $u_{0}$ is an initial approximation of equation (5), which satisfies the boundary conditions. Considering equation (8), we will have

$$
\begin{array}{ll}
H(v, 0)=L(v)-L\left(u_{0}\right)=0 \\
\text { and } \quad & H(v, 1)=A(v)-f(r)=0
\end{array}
$$

The changing process of $p$ from zero to unity is just that of $v(r, p)$ from $u_{0}(r)$ to $u(r)$. In topology this is called deformation and $L(v)-L\left(u_{0}\right)$ and $A(v)-f(r)$ are called homotopy.

According to the homotopy perturbation theory, we can first use the embedding parameter $p$ as a small parameter and assume that the solution of equation (8) can be written as a power series in $p$ as follows:

$$
v=v_{0}+p v_{1}+p^{2} v_{2}+p^{3} v_{3}+
$$

To obtain the approximate solution of equation (1) setting $p=1$, we have

$$
u=\lim _{p \rightarrow 1} v=v_{0}+v_{1}+v_{2}+v_{3}+\ldots \ldots \ldots \ldots \ldots . . .
$$

The equation (12) is convergent for most cases. However, the convergent rate depends on the nonlinear operator $A(v)$. 
Asymptotic Solutions of Coupled Spring Systems with Cubic Nonlinearity using Homotopy Perturbation Method

\section{Application of the Homotopy Perturbation Method (HPM)}

Writing $x$ for $x_{1}$ and $y$ for $x_{2}$ we may rewrite the equations (3) and (4) as follow:

$$
\begin{aligned}
& m_{1} x^{\prime \prime}=-\delta_{1} x^{\prime}-k_{1} x-k_{2}(x-y)+\mu_{1} x^{3}+\mu_{2}(x-y)^{3}+F_{1} \cos \omega_{1} t \\
& m_{2} y^{\prime \prime}=-\delta_{2} y^{\prime}-k_{2}(y-x)+\mu_{2}(y-x)^{3}+F_{2} \cos \omega_{2} t
\end{aligned}
$$

and (14) are

According to the equation (8), we consider the following homotopy for the (13)

$$
\begin{aligned}
& m_{1} x^{\prime \prime}+\delta_{1} x^{\prime}+k_{1} x-k_{2}(y-x)+p\left[-\mu_{1} x^{3}+\mu_{2}(y-x)^{3}-F_{1} \cos \omega_{1} t\right]=0 \\
& m_{2} y^{\prime \prime}+\delta_{2} y^{\prime}+k_{2}(y-x)+p\left[-\mu_{2}(y-x)^{3}-F_{2} \cos \omega_{2} t\right]=0
\end{aligned}
$$

As outlined above, the basic assumption is that the solutions of equations (13) and (14) can be written as power series in $p$ :

$$
\begin{aligned}
& x=x_{0}+p x_{1}+p^{2} x_{2}+. \\
& y=y_{0}+p y_{1}+p^{2} y_{2}+.
\end{aligned}
$$

Therefore, substituting (17) and (18) into (15) and (16), and then equating the terms with identical powers of $p$, we can obtain the following set of linear differential equations:

$$
\begin{aligned}
& p^{0}:\left\{\begin{array}{l}
k_{1} x_{0}+k_{2} x_{0}-k_{2} y_{0}+\delta_{1} x_{0}^{\prime}+m_{1} x_{0}^{\prime \prime}=0 \\
-k_{2} x_{0}+k_{2} y_{0}+\delta_{2} y_{0}^{\prime}+m_{2} y_{0}^{\prime \prime}=0
\end{array}\right. \\
& p^{1}:\left\{\begin{array}{lr}
-F_{1} \cos t \omega_{1}-\mu_{1} x_{0}{ }^{3}-\mu_{2} x_{0}{ }^{3}+k_{1} x_{1}+k_{2} x_{1}+3 \mu_{2} x_{0}{ }^{2} y_{0}-3 \mu_{2} x_{0} y_{0}{ }^{2} \\
+\mu_{2} y_{0}{ }^{3}-k_{2} y_{1}+\delta_{1} x_{1}^{\prime}+m_{1} x_{1}^{\prime \prime}=0 & x_{1}(0)=0, x_{1}^{\prime}(0)=0 \\
-F_{2} \cos t \omega_{2}+\mu_{2} x_{0}{ }^{3}-k_{2} x_{1}-3 \mu_{2} x_{0}{ }^{2} y_{0}+3 \mu_{2} x_{0} y_{0}{ }^{2}-\mu_{2} y_{0}{ }^{3}+k_{2} y_{1} \\
+\delta_{2} y_{1}^{\prime}+m_{2} y_{1}^{\prime \prime}=0 & y_{1}(0)=0, y_{1}^{\prime}(0)=0
\end{array}\right.
\end{aligned}
$$

Case I (undamped undriven motion): In the absence of damping and external driving force, i.e., $F_{1}=0, F_{2}=0, \delta_{1}=0, \delta_{2}=0$, we choose $m_{1}=m_{2}=1, \quad k_{1}=0.4, \quad k_{2}=1.808$, $\mu_{1}=-0.16, \mu_{2}=-0.1$. Then subject to the initial conditions $x(0)=0.005, y(0)=0.001$, the first few approximations of the homotopy perturbation solutions for equations (13) and (14) are derived in the following forms:

$$
\begin{aligned}
& x_{0}=0.0027221 e^{2.602 \times 10^{-18} t} \cos 0.435 t+0.0022779 \cos 1.956 t \\
& y_{0}=0.00303982 e^{2.602 \times 10^{-18} t} \cos 0.435 t-0.00203982 \cos 1.956 t
\end{aligned}
$$


Md. Abdul Alim, M. Abul Kawser and Md. Mizanur Rahman

$$
\begin{aligned}
x_{1}= & \left(-2.95646 \times 10^{-7} e^{2.602 \times 10^{-18} t}+2.93066 \times 10^{-7} e^{7.806 \times 10^{-18} t}\right) \cos 0.435 t \\
+ & \left(7.57125-7.43861 e^{5.204 \times 10^{-18} t}\right) \times 10^{-8} \cos 1.956 t+\left\{2.38093 \times 10^{8}\right. \\
& \left.\left(e^{2.602 \times 10^{-18} t}-\mathrm{e}^{7.806 \times 10^{-18} t}\right)-1.78825 \times 10^{-9} e^{2.602 \times 10^{-18} t} t\right\} \sin 0.4347 t \\
& +\left\{1.13789 \times 10^{8}\left(1-e^{5.204 \times 10^{-18} t}\right)-1.82433 \times 10^{-9} t\right\} \sin 1.956 t \\
y_{1}= & \left(-3.11678 \times 10^{-7} e^{2.602 \times 10^{-18} t}+3.13196 \times 10^{-7} e^{7.806 \times 10^{-18} t}\right) \cos 0.4347 t \\
+ & 1.38875 \times 10^{-9} e^{5.204 \times 10^{-18} t} \cos 1.08686 t-1.14846 \times 10^{-9} e^{2.602 \times 10^{-18} t} \cos 1.304 t \\
- & 1.10911 \times 10^{-9} \cos 1.956 t+\left\{2.65883 \times 10^{8}\left(e^{2.602 \times 10^{-18} t}-e^{7.806 \times 10^{-18} t}\right)\right. \\
- & \left.1.99697 \times 10^{-9} e^{2.602 \times 10^{-18} t} t\right\} \sin 0.435 t-\left\{1.01896 \times 10^{8}\left(1-e^{5.204 \times 10^{-18} t}\right)\right. \\
- & \left.1.63365 \times 10^{-9} t\right\} \sin 1.956 t
\end{aligned}
$$

Therefore, the solutions up to first approximations of the equations (13) and (14) are $x=x_{0}+x_{1}$ and $y=y_{0}+y_{1}$

Table 1: Comparison between the HMP and Numerical results for $x(t)$ and $y(\mathrm{t})$

\begin{tabular}{|c|c|c|c|c|c|c|}
\hline \multirow{2}{*}{$\begin{array}{c}\text { Time } \\
(\mathbf{t})\end{array}$} & \multicolumn{2}{|c|}{ Homotopy Results } & \multicolumn{2}{c|}{ Numerical Results } & \multicolumn{2}{c|}{ Errors in \% } \\
\cline { 2 - 7 } & $x(t)$ & $y(\mathrm{t})$ & $x(t)$ & $y(\mathrm{t})$ & $x(t)$ & $y(\mathrm{t})$ \\
\hline 0 & 0.005 & 0.001 & 0.005 & 0.001 & 0 & 0 \\
\hline 4 & -0.00038953 & -0.0005673 & -0.00038956 & -0.00056727 & 0.009851 & 0.00474139 \\
\hline 8 & -0.00484392 & -0.00083333 & -0.00484375 & -0.00083347 & 0.0033871 & 0.0166006 \\
\hline 12 & 0.00111795 & 0.00164481 & 0.0011179 & 0.00164477 & 0.0044629 & 0.00222489 \\
\hline 16 & 0.00439269 & 0.00035231 & 0.00439243 & 0.00035257 & 0.0057609 & 0.0729195 \\
\hline 20 & -0.00169985 & -0.00255738 & -0.0016998 & -0.00255735 & 0.0025688 & 0.00095026 \\
\hline 24 & -0.00369565 & 0.00038805 & -0.00369523 & 0.00038772 & 0.0114081 & 0.0840284 \\
\hline 28 & 0.00205594 & 0.00321559 & 0.00205596 & 0.00321561 & 0.0011179 & 0.00054631 \\
\hline 32 & 0.00282861 & -0.00130321 & 0.00282813 & -0.00130275 & 0.0168171 & 0.0356236 \\
\hline 36 & -0.00213242 & -0.00355852 & -0.00213246 & -0.00355848 & 0.0017270 & 0.00118794 \\
\hline 40 & -0.00188585 & 0.00228806 & -0.00188525 & 0.00228751 & 0.0318059 & 0.0240745 \\
\hline
\end{tabular}

This fact is graphically shown in Figure 2.

Case II (damped undriven motion): In the absence of the external forces, that is $F_{1}=0, F_{2}=0$, we choose $m_{1}=1, m_{2}=1, k_{1}=3, k_{2}=2, \delta_{1}=0.1, \delta_{2}=0.2, \mu_{1}=0.16$, $\mu_{2}=0.1$. Then subject to the initial conditions $x(0)=0.6, y(0)=0.2$, the first few approximations of the homotopy perturbation solutions for equations (13) and (14) are derived in the following forms:

$$
\begin{aligned}
x_{0}= & 0.199706 e^{-0.09 t} \cos 0.996 t+0.400294 e^{-0.06 t} \cos 2.448 t \\
& +0.005252 e^{-0.09 t} \sin 0.996 t+0.015012 e^{-0.06 t} \sin 2.448 t \\
y_{0}= & 0.399891 e^{-0.09 t} \cos 0.996 t-0.199891 e^{-0.06 t} \cos 2.448 t \\
& +0.01848 e^{-0.09 t} \sin 0.996 t+0.002288 e^{-0.06 t} \sin 2.448 t
\end{aligned}
$$


Asymptotic Solutions of Coupled Spring Systems with Cubic Nonlinearity using Homotopy Perturbation Method

$$
\begin{aligned}
x_{1} & =0.000636 e^{-0.24 t} \cos 0.456 t+\left(0.000051 e^{-0.27 t}+0.000199 e^{-0.21 t}\right. \\
& \left.-0.001828 e^{-0.09 t}\right) \cos 0.996 t+\left(0.000628 e^{-0.24 t}+0.002815 e^{-0.18 t}\right. \\
& \left.-0.002431 e^{-0.06 t}\right) \cos 2.448 t-0.000051 e^{-0.27 t} \cos 2.988 t \\
& +0.000255 e^{-0.21 t} \cos 3.901 t-0.000268 e^{-0.24 t} \cos 4.441 t \\
& +0.000049 e^{-0.21 t} \cos 5.893-0.000177 e^{-0.18 t} \cos 7.345 t \\
& -0.000071 e^{-0.24 t} \sin 0.456 t-\left(0.000869 e^{-0.27 t}+0.01538 e^{-0.21 t}\right. \\
& \left.-0.016485 e^{-0.09 t}\right) \sin 0.996 t-\left(0.008413 e^{-0.24 t}+0.043656 e^{-0.18 t}\right. \\
& \left.-0.052217 e^{-0.06 t}\right) \sin 2.448-0.000071 e^{-0.24 t} \sin 4.441 t \\
y_{1} & =-0.000268 e^{-0.24 t} \cos 0.456 t+\left(0.000156 e^{-0.27 t}+0.004426 e^{-0.21 t}\right. \\
& \left.-0.00432 e^{-0.09 t}\right) \cos 0.996 t-\left(0.000154 e^{-0.24 t}-0.000505 e^{-0.18 t}\right) \cos 2.448 t \\
& -0.000051 e^{-0.27 t} \cos 2.988 t-0.000502 e^{-0.21 t} \cos 3.9 t+0.000084 e^{-0.24 t} \\
& \cos 4.44 t-0.00021 e^{-0.21 t} \cos 5.893 t+0.000178 e^{-0.18 t} \cos 7.345 t- \\
& \left(0.001741 e^{-0.27 t}+0.030824 e^{-0.21 t}-0.032955 e^{-0.09 t}\right) \sin 0.996 t \\
& +\left(0.004201 e^{-0.24 t}+0.021965 e^{-0.18 t}-0.026086 e^{-0.06 t}\right) \sin 2.448 t \\
& \text { Therefore, the solutions up to first approximations of the equations }(13) \text { and }(14) \text { are } \\
& x=x_{0}+x_{1} \text { and } y=y_{0}+y_{1}
\end{aligned}
$$

Table 2: Comparison between the HPM and Numerical results for $x(t)$ and $y(\mathrm{t})$

\begin{tabular}{|c|c|c|c|c|c|c|}
\hline \multirow{2}{*}{ Time (t) } & \multicolumn{2}{|c|}{ Homotopy Results } & \multicolumn{2}{c|}{ Numerical Results } & \multicolumn{2}{c|}{ Errors in \% } \\
\cline { 2 - 7 } & $x(t)$ & $y(\mathrm{t})$ & $x(t)$ & $y(\mathrm{t})$ & $x(t)$ & $y(\mathrm{t})$ \\
\hline 0 & 0.6 & 0.2 & 0.6 & 0.2 & 0 & 0 \\
\hline 4 & -0.401964 & -0.0522376 & -0.401495 & -0.0523665 & 0.116662 & 0.246265 \\
\hline 8 & 0.199569 & -0.100768 & 0.198919 & -0.100408 & 0.327039 & 0.358525 \\
\hline 12 & -0.0598972 & 0.153063 & -0.0595017 & 0.152605 & 0.664585 & 0.300552 \\
\hline 16 & -0.0090066 & -0.10902 & -0.00904281 & -0.108719 & 0.400482 & 0.276927 \\
\hline 20 & 0.0325736 & 0.028401 & 0.0323433 & 0.0283948 & 0.712161 & 0.0217638 \\
\hline 24 & -0.0387334 & 0.0340943 & -0.0383576 & 0.0338563 & 0.979746 & 0.702848 \\
\hline 28 & 0.0422335 & -0.0572848 & 0.0417994 & -0.0569481 & 1.03853 & 0.591232 \\
\hline 32 & -0.0449839 & 0.0492994 & -0.0445549 & 0.0489943 & 0.962987 & 0.622844 \\
\hline 36 & 0.043708 & -0.0292358 & 0.0433348 & -0.0290241 & 0.861144 & 0.729234 \\
\hline 40 & -0.0364871 & 0.0119506 & -0.0362054 & 0.0118327 & 0.777912 & 0.99628 \\
\hline
\end{tabular}

This fact is graphically shown in Figure 3.

Case III (undamped driven motion): In the absence of the damping, that is $\delta_{1}=0$, $\delta_{2}=0$, we choose $m_{1}=1, m_{2}=1, k_{1}=0.4, k_{2}=0.5, \mu_{1}=-0.16, \mu_{2}=-0.1 \quad F_{1}=0.005$, $F_{2}=0.003, \omega_{1}=0.5, \omega_{2}=0.4$. Then subject to the initial conditions $x(0)=0.005$, 
Md. Abdul Alim, M. Abul Kawser and Md. Mizanur Rahman

$y(0)=0.001$, the first few approximations of the homotopy perturbation solutions for equations (13) and (14) are derived in the following form:

$$
\begin{aligned}
x_{0} & =0.00203576 \cos 0.402 t+0.00296424 \cos 1.113 t \\
y_{0} & =0.00300689 \cos 0.402 t-0.00200689 \cos 1.113 t \\
x_{1} & =(-7.48048 \cos 0.309 t-3.1403 \cos 0.402 t-4.04155 \cos 1.113 t \\
& +1.12146 \cos 1.206 t-2.75918 t \sin 0.402 t-6.99461 t \sin 1.113 t) \times 10^{-9} \\
& +0.9375 \cos 0.4 t-0.0142857 \cos 0.5 t+(7.02903 \cos 1.917 t \\
& +4.2854 \cos 3.339 t) \times 10^{-10} \\
y_{1} & =(-8.38175 \cos 0.309 t-5.8168 \cos 0.402 t-4.07541 t \sin 0.402 t \\
& +4.73558 t \sin 1.113 t) \times 10^{-9}+1.3875 \cos 0.4 t-0.028571 \cos 0.5 t \\
& +0.00147389 \cos 1.113 t+(6.9283 \cos 1.824 t-5.47984 \cos 1.206 t \\
& -2.13792 \cos 1.917 t+3.25223 \cos 2.628 t-2.90135 \cos 3.339 t) \times 10^{-10}
\end{aligned}
$$

Therefore, the solutions up to first approximation of the equations (13) and (14) are

$$
x=x_{0}+x_{1} \text { and } y=y_{0}+y_{1}
$$

Table 3: Comparison between the HPM and Numerical results for $x(t)$ and $y(\mathrm{t})$

\begin{tabular}{|c|c|c|c|c|c|c|}
\hline \multirow{2}{*}{$\begin{array}{c}\text { Time } \\
(\mathbf{t})\end{array}$} & \multicolumn{2}{|c|}{ Homotopy Results } & \multicolumn{2}{c|}{ Numerical Results } & \multicolumn{2}{c|}{ Errors in \% } \\
\cline { 2 - 7 } & $x(t)$ & $y(\mathrm{t})$ & $x(t)$ & $y(\mathrm{t})$ & $x(t)$ & $y(\mathrm{t})$ \\
\hline 0 & 0.005 & 0.001 & 0.005 & 0.001 & 0 & 0 \\
\hline 4 & 0.0119992 & 0.0211882 & 0.011999 & 0.0211881 & 0.00113002 & 0.0005929 \\
\hline 8 & -0.0107062 & -0.0122366 & -0.0107067 & -0.0122379 & 0.00441015 & 0.0103707 \\
\hline 12 & -0.0318465 & -0.0551639 & -0.0318378 & -0.0551574 & 0.0271398 & 0.0117505 \\
\hline 16 & 0.0244173 & 0.0390611 & 0.0244297 & 0.0390838 & 0.0506395 & 0.0579618 \\
\hline 20 & 0.0420799 & 0.0696532 & 0.0420325 & 0.0696118 & 0.112767 & 0.0594703 \\
\hline 24 & -0.0382782 & -0.0655714 & -0.0383281 & -0.0656552 & 0.13031 & 0.12754 \\
\hline 28 & -0.0437812 & -0.0667641 & -0.0436527 & -0.0666414 & 0.294185 & 0.184104 \\
\hline 32 & 0.0454436 & 0.0778186 & 0.04555 & 0.0779881 & 0.23342 & 0.217232 \\
\hline 36 & 0.0437333 & 0.0601494 & 0.0434981 & 0.0599155 & 0.540698 & 0.390225 \\
\hline 40 & -0.0448386 & -0.0734008 & -0.0449987 & -0.0736595 & 0.35578 & 0.35129 \\
\hline
\end{tabular}

This fact is graphically shown in the Figure 4.

Case IV (damped driven motion): In the presence of both damping and external forces we choose $m_{1}=1, \quad m_{2}=1, \quad \delta_{1}=0.2, \quad \delta_{2}=0.3, \quad k_{1}=6, \quad k_{2}=4, \quad \mu_{1}=0.16, \quad \mu_{2}=0.1$, $F_{1}=0.3, \quad F_{2}=0.2, \omega_{1}=1, \omega_{2}=0.6$. Then subject to the initial conditions $x(0)=0.7$, $y(0)=0.2$, the first few approximations of the homotopy perturbation solutions for the equations (13) and (14) are derived in the following form:

$$
\begin{aligned}
x_{0} & =0.219825 e^{-0.14 t} \cos 1.407 t+0.480175 e^{-0.11 t} \cos 3.462 t \\
& +0.011219 e^{-0.14 t} \sin 1.407 t+0.019584 e^{-0.11 t} \sin 3.462 t
\end{aligned}
$$


Asymptotic Solutions of Coupled Spring Systems with Cubic Nonlinearity using Homotopy Perturbation Method

$$
\begin{aligned}
y_{0}= & 0.439932 e^{-0.14 t} \cos 1.407 t-0.239932 e^{-0.11 t} \cos 3.462 t \\
& +0.028659 e^{-0.14 t} \sin 1.407 t-0.001479 e^{-0.11 t} \sin 3.462 t \\
x_{1}= & 0.041418 \cos 0.6 t+0.000461 e^{-0.39 t} \cos 0.647 t+0.07768 \cos t+ \\
& \left(0.000237 e^{-0.36 t}-0.1057 e^{-0.14 t}\right) \cos 1.407 t+\left(0.000453 e^{-0.39 t}+\right. \\
& \left.0.0022885 e^{-0.33 t}-0.01683 e^{-0.11 t}\right) \cos 3.462 t+0.0001995 e^{-0.36 t} \cos 5.516 t \\
& -0.000193 e^{-0.39 t} \cos 6.277 t+0.000038 e^{-0.36 t} \cos 8.332 t-0.000153 e^{-0.33 t} \\
& \cos 10.386 t+0.004771 \sin 0.6 t-0.000064 e^{-0.39 t} \sin 0.647 t+ \\
& 0.0149435 \sin t-\left(0.000528 e^{-0.42 t}+0.009359 e^{-0.36 t}+0.0117 e^{-0.14 t}\right) \\
& \sin 1.407 t-\left(0.005563 e^{-0.39 t}+0.029079 e^{-0.33 t}-0.033743 e^{-0.11 t}\right) \sin 3.462 t \\
& -0.000131 \sin 5.924 t-0.000064 e^{-0.39 t} \sin 6.277 t+0.000131 \sin 7.924 t \\
y_{1} & =0.099959 \cos 0.6 t-0.000193 e^{-0.39 t} \cos 0.647 t+0.100541 \cos t \\
& +\left(0.000069 e^{-0.42 t}+0.003435 e^{-0.36 t}-0.211357 e^{-0.14 t}\right) \cos 1.407 t \\
& -\left(0.000112 e^{-0.39 t}-0.000116 e^{-0.33 t}-0.007815 e^{-0.11 t}\right) \cos 3.462 t \\
& -0.000069 e^{-0.42 t} \cos 4.222 t-0.000398 e^{-0.36 t} \cos 5.516 t \\
& +0.00006 e^{-0.39 t} \cos 6.277 t-0.000167 e^{-0.36 t} \cos 8.332 t \\
& +0.000153 e^{-0.33 t} \cos 10.386 t+0.010121 \sin 0.6 t+0.030327 \sin t \\
& -\left(0.001057 e^{-0.42 t}+0.018746 e^{-0.36 t}+0.026408 e^{-0.14 t}\right) \sin 1.407 t \\
+ & \left(0.00278 e^{-0.39 t}+0.014681 e^{-0.33 t}-0.017128 e^{-0.11 t}\right) \sin 3.462 t
\end{aligned}
$$

Therefore, the solutions up to first approximation of the equations (13) and (14) are $x=x_{0}+x_{1}$ and $y=y_{0}+y_{1}$

Table 4: Comparison between HPM and Numerical results for $x(t)$ and $y(\mathrm{t})$

\begin{tabular}{|c|c|c|c|c|c|c|}
\hline \multirow{2}{*}{$\begin{array}{c}\text { Time } \\
(\mathbf{t})\end{array}$} & \multicolumn{2}{|c|}{ Homotopy Results } & \multicolumn{2}{c|}{ Numerical Results } & \multicolumn{2}{c|}{ Errors in \% } \\
\cline { 2 - 7 } & $x(t)$ & $y(\mathrm{t})$ & $x(t)$ & $y(\mathrm{t})$ & $x(t)$ & $y(\mathrm{t})$ \\
\hline 0 & 0.7 & 0.2 & 0.7 & 0.2 & 0 & 0 \\
\hline 4 & 0.0732052 & -0.0988329 & 0.0715742 & -0.0986097 & 2.27881 & 0.226382 \\
\hline 8 & -0.137313 & 0.110924 & -0.136801 & 0.113806 & 0.37374 & 2.53262 \\
\hline 12 & -0.0247882 & 0.171342 & -0.0228239 & 0.172811 & 8.6065 & 0.84995 \\
\hline 16 & -0.106899 & -0.2396 & -0.1062 & -0.239424 & 0.658007 & 0.0735566 \\
\hline 20 & 0.122812 & 0.108081 & 0.122675 & 0.108229 & 0.111443 & 0.136858 \\
\hline 24 & 0.0192406 & -0.0105786 & 0.0189694 & -0.0106785 & 1.42985 & 0.935364 \\
\hline 28 & -0.112351 & -0.134943 & -0.112441 & -0.135137 & 0.0802358 & 0.143812 \\
\hline 32 & 0.103921 & 0.204301 & 0.103981 & 0.204273 & 0.0585195 & 0.0136497 \\
\hline 36 & -0.0571445 & -0.132104 & -0.0571877 & -0.132158 & 0.0755187 & 0.0405423 \\
\hline 40 & -0.0210969 & -0.0132315 & -0.0211216 & -0.0132385 & 0.116604 & 0.05299 \\
\hline
\end{tabular}

This fact is graphically shown in Figure 5. 
Md. Abdul Alim, M. Abul Kawser and Md. Mizanur Rahman

\section{Result and discussion}

The homotopy perturbation method is successfully applied to solve the nonlinear ordinary differential equations governing the motion of a coupled spring system. To test the accuracy of the HPM results, we match our results with the numerical results obtained by using Mathematica 9.0. The solution obtained by Homotopy perturbation method is an infinite series for appropriate initial conditions. Reasonably appropriate values for the spring constants $k_{1}, k_{2}$, damping constants $\delta_{1}, \delta_{2}$, nonlinear constants $\mu_{1}, \mu_{2}$, forcing constant $F_{1}, F_{2}$ and $\omega_{1}, \omega_{2}$ are taken for computing HPM solutions. Comparison between these and the corresponding numerical solutions computed by the Mathematica 9.0 program for various values of $t$ are shown in the figures from Figure 2 to Figure 5 respectively in all four cases we study. These solutions represent a wide variety of interesting motions.

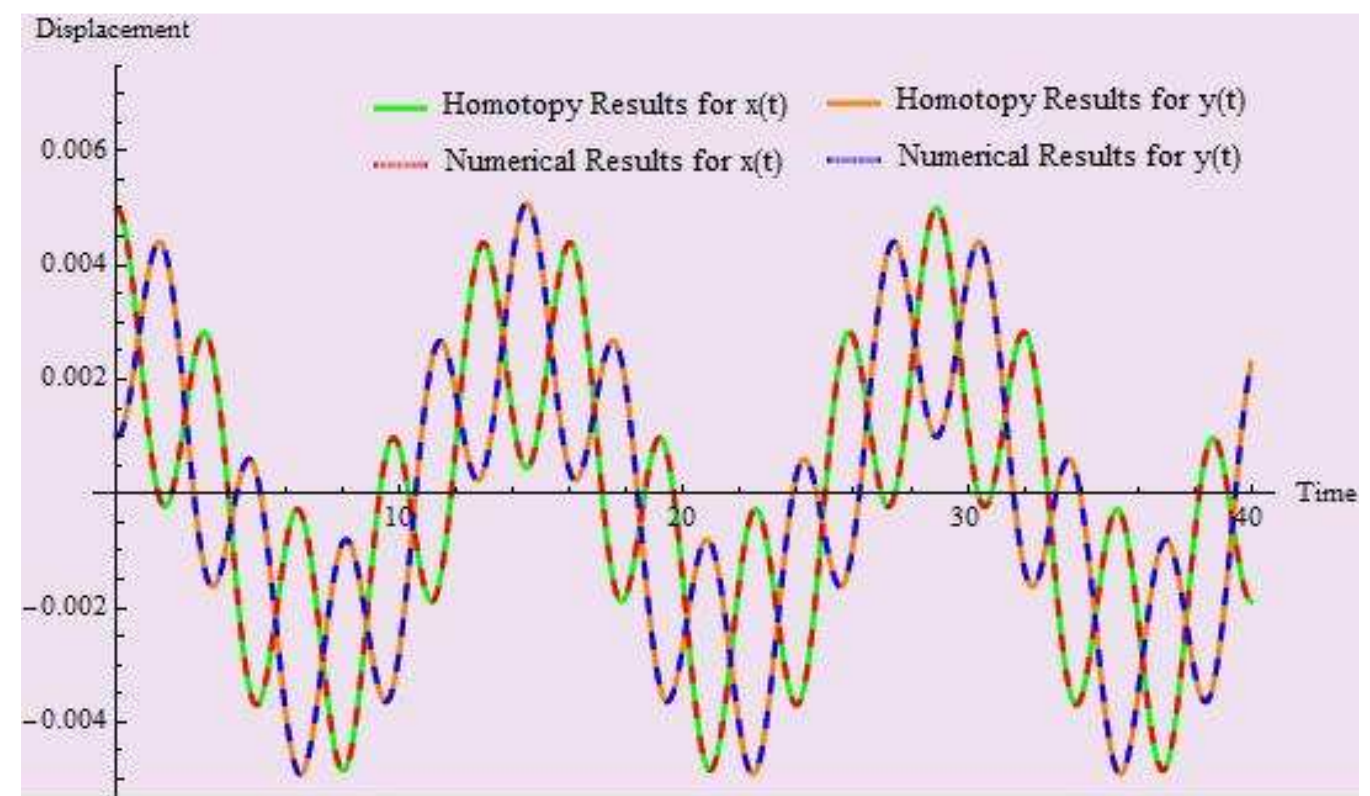

Figure 2: Comparison for $F_{1}=0, F_{2}=0, \delta_{1}=0, \delta_{2}=0, m_{1}=1, m_{2}=1, k_{1}=0.4$, $k_{2}=1.808, \mu_{1}=-0.16, \mu_{2}=-0.1$ and the initial conditions $x(0)=0.005, y(0)=0.001$. 
Asymptotic Solutions of Coupled Spring Systems with Cubic Nonlinearity using Homotopy Perturbation Method

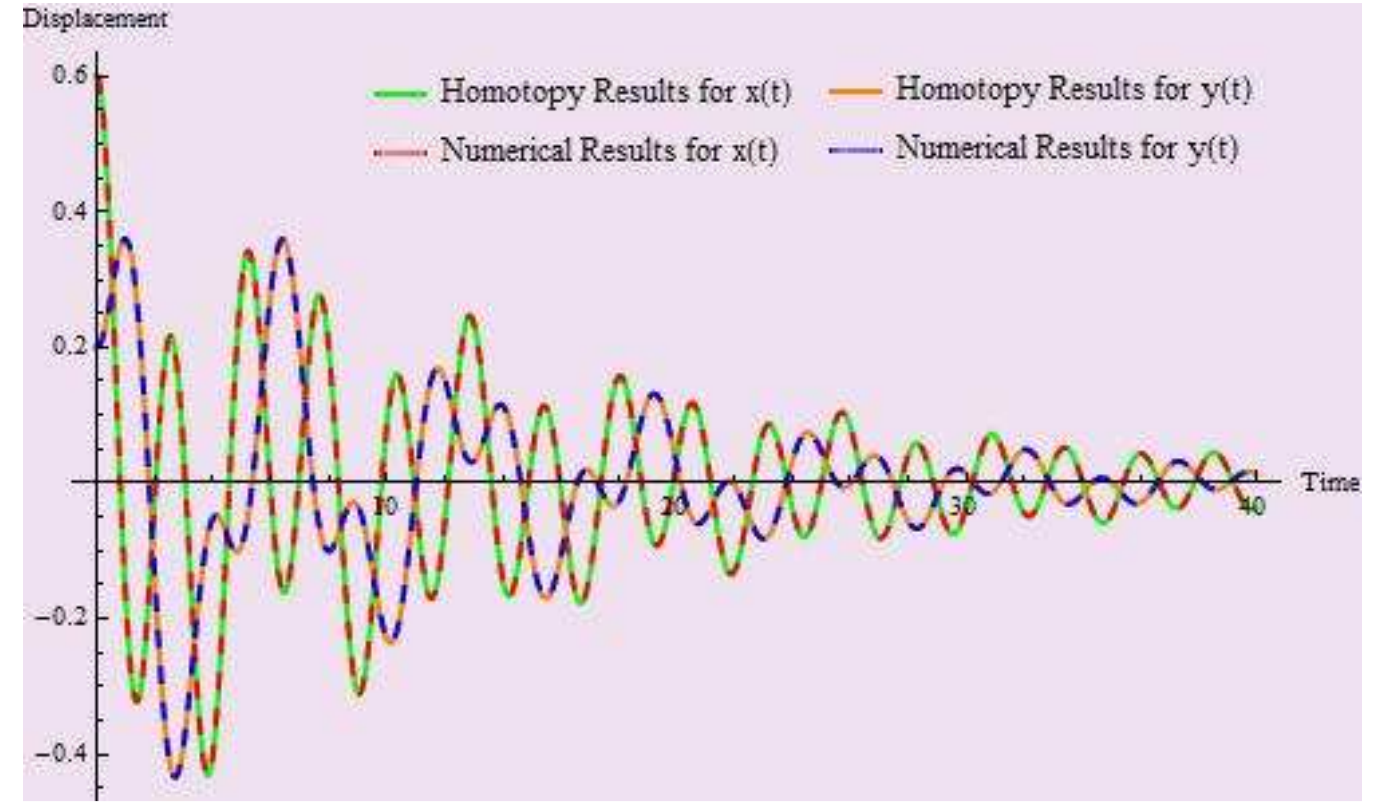

Figure 3: Comparison for $F_{1}=0, F_{2}=0, \delta_{1}=0.1, \delta_{2}=0.2, m_{1}=1, m_{2}=1, k_{1}=3$, $k_{2}=2, \mu_{1}=0.16, \mu_{2}=0.1$ and the initial conditions $x(0)=0.6, y(0)=0.2$.

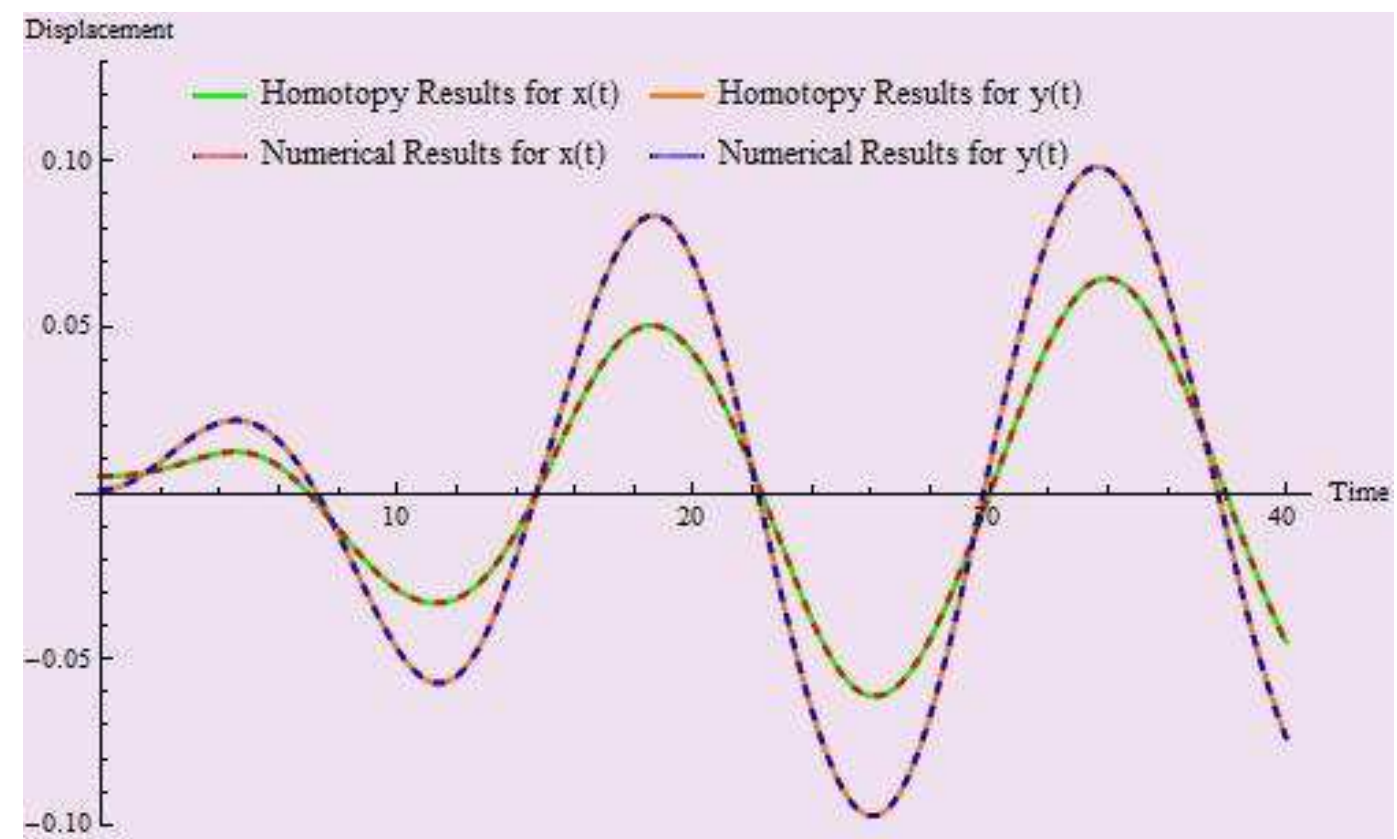

Figure 4: Comparison for $F_{1}=0.005, F_{2}=0.003, \delta_{1}=0, \delta_{2}=0, m_{1}=1, m_{2}=1, k_{1}=0.4$, $k_{2}=0.5, \mu_{1}=-0.16, \mu_{2}=-0.1, \omega_{1}=0.5, \omega_{2}=0.4$ and the initial conditions $x(0)=0.005$, $y(0)=0.001$. 
Md. Abdul Alim, M. Abul Kawser and Md. Mizanur Rahman

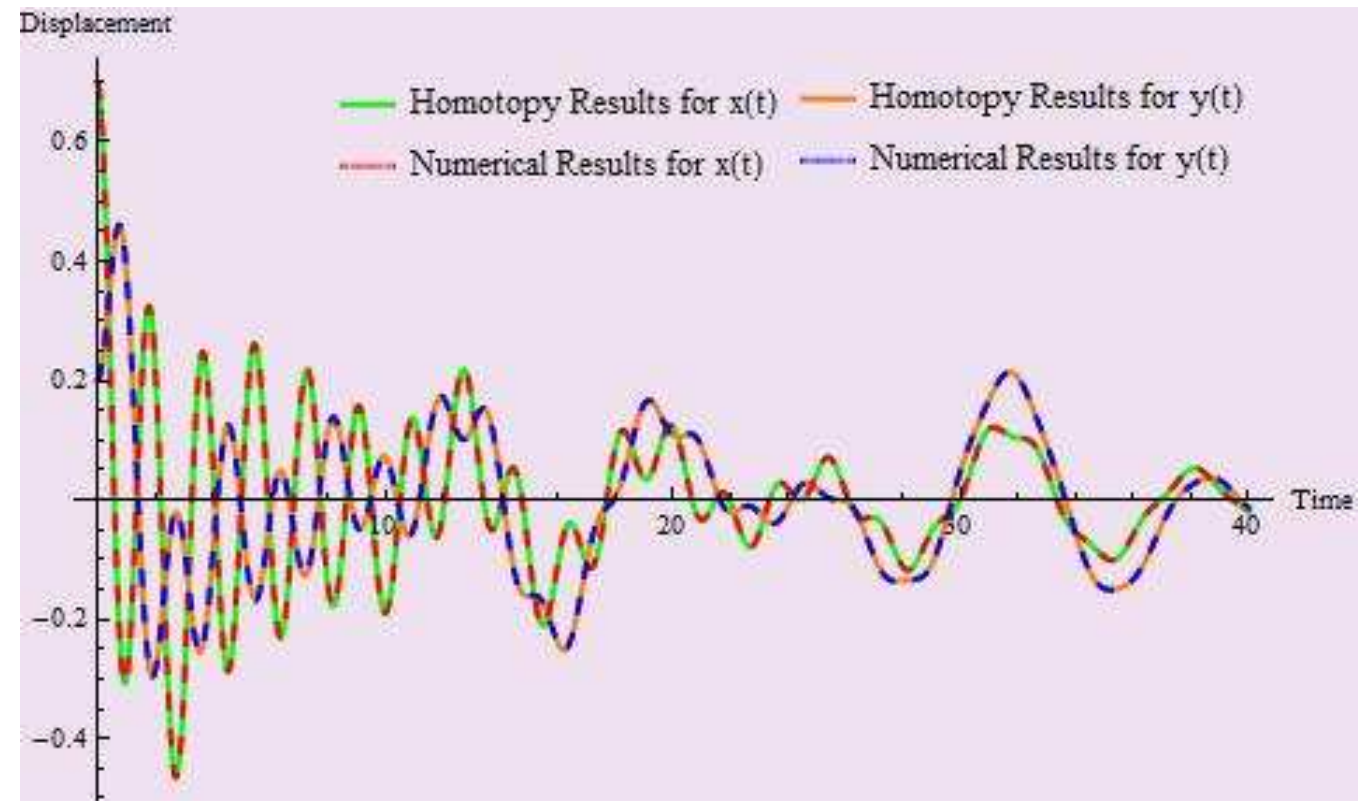

Figure 5: Comparison for $F_{1}=0.3, F_{2}=0.2, \delta_{1}=0.2, \delta_{2}=0.3, m_{1}=1, m_{2}=1, k_{1}=6$, $k_{2}=4, \mu_{1}=0.16, \mu_{2}=0.1, \omega_{1}=1, \omega_{2}=0.6$ and the initial conditions $x(0)=0.7$, $y(0)=0.2$.

\section{Conclusion}

In this article, the homotopy perturbation method (HPM) has been successfully applied to solve the dynamics of a coupled spring system with cubic nonlinearity. Our results suggest that it is an efficient method for obtaining solutions of nonlinear differential equations governing the motion of such problems. They also confirm the simplicity and efficiency of the method for solving any nonlinear ordinary differential equation or systems of nonlinear ordinary differential equations. It is also observed that the HPM is a promising method for solving other linear and nonlinear partial differential equations.

Acknowledgements: This research is supported by Islamic University, Kushtia, Bangladesh. We like to thank all honourable members of the Academic Committee of the Department of Mathematics for providing us with logistic supports in carrying out the research. We also gratefully thank the anonymous reviewers for their valuable comments which helped us improve the manuscript significantly.

\section{REFERENCES}

1. J.H.He, New interpretation of homotopy perturbation method, International Journal of Modern Physics B, 20 (2006) 2561-2568.

2. J.H.He, Some asymptotic methods for strongly nonlinear equations, International Journal of Modern Physics B, 20 (2006) 1141-1199.

3. D.H.Shou, The homotopy perturbation method for nonlinear oscillators, Computers \&Mathematics with Applications, 58 (2009) 2456-2459. 
Asymptotic Solutions of Coupled Spring Systems with Cubic Nonlinearity using Homotopy Perturbation Method

4. J.H.He, Homotopy perturbation technique, Computer Methods in Applied Mechanics and Engineering, 178 (1999) 257-262.

5. J.H.He, New perturbation technique which is also valid for large parameters, Journal of Sound and Vibration, 229 (2000) 1257-1263.

6. J.H.He, A coupling method of homotopy technique and perturbation technique for nonlinear problems, International Journal of Non-Linear Mechanics, 35 (2000) 37-43.

7. J.H.He, Homotopy Perturbation Technique. Computer Methods in Applied Mechanics and Engineering, 178 (1999) 257-262.

8. J.H.He, Variational iteration method-some recent results and new interpretations. Journal of Computational and Applied Mathematics, 207 (2007) 3-17.

9. D.D.Ganji, H.Tari, and M.B.Jooybari, Variational iteration method and homotopy perturbation method for nonlinear evolution equations, Computers \& Mathematics with Applications, 54 (2007) 1018-1027.

10. J.H.He, and X.H.Wu, Construction of solitary solution and compacton-like solution by variational iteration method, Chaos Solitons \& Fractals, 29 (2006) 108-113.

11. A.R. Manwell, The Tricomi Equations with Applications to the Theory of Plane Transonic Flow, Pitman, London, UK 1979

12. J.Smollet, Shock Waves and Reaction Diffusion Equations, Springer Verlag, New York 1983.

13. J.H.He, Homotopy perturbation method for solving boundary value problem, Physics Letters A, 350 (2006) 87-96.

14. J.H.He, A simple perturbation approach to blasius equation, Applied Mathematics and Computation, 140 (2003) 217-222.

15. D.D.Ganji and M.Rafei, Solitary wave solutions for a generalisezed hirota-satsuma coupled kdv equations by homotopy perturbation method, Physics Letters A, 356 (2006) 131-137.

16. A.Sadighi and D.D.Ganji, Exact solutions of laplace equation by homotopy perturbation andadomian decomposition methods, Phys. Lett. A, 367 (2007) 83-87.

17. D.D.Ganji and A.Rajabi, Assessment of homotopy perturbation and perturbation methods in heat radiation equations, Int. Commun. Heat Mass Transf., 33 (2006) 391-400.

18. G.Domairry, M.Ahangari and M.Jamshidi, Exact and analytical solution for nonlinear dispersive $\mathrm{k}(\mathrm{mp})$ equations using homotopy perturbation method, Phys. Lett. A, 368 (2007) 266-270.

19. D.D.Ganji, G.A.Afrouzi, H.Hosseinzadeh and R.A.Talarposhti, Application of homotopy perturbation method to the second kind of nonlinear integral equations, Phys. Lett. A, 371 (2007) 20-25.

20. A.Rajabi, D.D.Ganji and H.Taherian, Application of homotopy perturbation method in nonlinear heat conduction and convection equations, Phys. Lett. A, 360 (2007) $570-573$.

21. A.Sadighi and D.D.Ganji, Analytic treatment of linear and nonlinear schrödinger equations: a study with homotopy perturbation and adomian decomposition methods, Phys. Lett. A, (2007).

22. J.H.He, The homotopy perturbation method for nonlinear oscillators with discontinuities, Appl. Math. Comput., 151 (2004) 287-292. 
Md. Abdul Alim, M. Abul Kawser and Md. Mizanur Rahman

23. J.H.He, Application of homotopy perturbation method to nonlinear wave equations, Chaos Solitons Fractals, 26 (2005) 695-700.

24. D.D.Ganji, M.Nourollahi and E.Mohseni, Application of He's methods to nonlinear chemistry problems, Comput. Math. Appl., 54 (2007) 1122-1132.

25. D.D.Ganji, The application of He's homotopy perturbation method to nonlinear equations arising in heat transfer, Phys. Lett. A, 355 (2006) 337-341.

26. M.Rafei, D.D.Ganji and H.Daniali, Solution of the epidemic model by homotopy perturbation method, Appl. Math. Comput., 187 (2007) 1056-1062.

27. J.H.He, Homotopy perturbation method: a new nonlinear analytic technique, Appl. Math. Comput., 135 (2003) 73-79.

28. J.H.He, Comparison of homotopy perturbation method and homotopy analysis method, Appl. Math. Comput., 156 (2004) 527-539.

29. J.H.He, Asymptotology by homotopy perturbation method, Appl. Math. Comput., 156 (2004) 591-596.

30. J.H.He, Limit cycle and bifurcation of nonlinear problems, Chaos Solitons Fractals, 26 (2005) 827-833.

31. J.H.He, Some asymptotic methods for strongly nonlinear equations, Int. J. Mod. Phys.B, 20 (2006) 1141-1199.

32. L.Cveticanin, The homotopy perturbation method applied for solving complex valued differential equations with strong cubic nonlinearity, J. Sound Vib., 285 (2005) 11711179.

33. T.H.Fay and D.G.Sarah, Coupled Spring Equations, Technikon Pretoria and Mathematics, University of Southern Mississippi, Box 5045, Hattiesburg, MS 394065045, USA, 2002

34. T.H.Fay, and S.V.Joubert, Energy and the nonsymmetric nonlinear spring, Int. J. Math. Educ. Sci. Technol., 30 (1999) 889-902.

35. R.Knapp and S.Wagon, Orbits worth betting on!, C_ODE_E Newsletter (1996) 9-13.

36. T.H.Fay and S.V.Joubert, Energy and contour plots for qualitative analysis of nonlinear differential equations, Math. Compute. Educ., 33 (1999) 67-77.

37. T.H.Fay and S.V.Joubert, Square waves from a black box, Math. Mag., 73 (2000) 393-399. 